\title{
Interfacial and surface energetics of $\mathrm{CoSi}_{2}$
}

\author{
D. P. Adams and S. M. Yalisove \\ Department of Materials Science and Engineering, University of Michigan, 2300 Hayward Street, \\ Ann Arbor, Michigan 48109-2136 \\ D. J. Eaglesham \\ AT\&T Bell Laboratories, Murray Hill, New Jersey 07974
}

(Received 23 March 1994; accepted for publication 20 July 1994)

The energetics of the $\mathrm{CoSi}_{2}$-Si interface and the $\mathrm{CoSi}_{2}$ surface have been investigated by analyzing the equilibrium shapes of isolated silicide precipitates. $\mathrm{CoSi}_{2}$ precipitates grown by heating $2 \AA$ of Co on a clean, reconstructed Si $\{100\}$ surface formed with a number of orientations that remained stable upon annealing to high temperatures. Precipitates buried by a Si capping layer were shown to form along $\{111\}$ and $\{100\}$ interfaces. A ratio of the $\mathrm{CoSi}_{2}$-Si interfacial free energies has been measured from the shapes of a large number of buried precipitates indicating that $\{100\} /\{111\}=1.43 \pm 0.07$. It is suggested that the shape of $\mathrm{CoSi}_{2}$ equilibrated within vacuum consists of $\{111\},\{100\}$, and $\{110\}$ facets.

\section{INTRODUCTION}

$\mathrm{CoSi}_{2}$ films are presently of interest as interconnects, contacts in very-large-scale integration, and for future threedimensional integration. ${ }^{1}$ This is due to its low resistivity $(\sim 1 \mu \Omega \mathrm{cm})$ and small lattice misfit to $\mathrm{Si}(-1.2 \%$ at room temperature). ${ }^{2}$ However, the usefulness of $\mathrm{CoSi}_{2}$ in many of these applications has become limited because current techniques do not produce layers of sufficient quality or stability. Regardless of the growth process used to build $\mathrm{CoSi}_{2}$ thin films, the energetics of the silicide-Si couple limits the integrity of the layer. This is especially true when a metal film is depositcd and annealed to high temperatures to form the silicide, e.g., in the self-aligned-silicide, or "salicide," process. ${ }^{3}$ This process becomes increasingly difficult to apply when very thin layers are desired, because the films exhibit grooving at grain boundaries and the continuous film breaks up into islands in an attempt to further reduce free energy. ${ }^{4}$ This islanding of silicides is one of the key limitations to a salicide process. In addition, novel growth techniques (such as the template technique) designed to make epitaxial structurcs with atomically abrupt interfaces, have been limited by the surface energetics of the silicide-Si couple. ${ }^{5}$ Progress in building such $\mathrm{CoSi}_{2}$-Si heterostructures has been slowed by the inability to grow $\mathrm{Si}$ on top of $\mathrm{CoSi}_{2}(100){ }^{6}$ The large interface energy of this system forces deposited $\mathrm{Si}$ to island, or ball up on the $\mathrm{CoSi}_{2}$, leading to a rough interface. It has therefore become crucial to understand the relative roles of each mechanism responsible for inhibiting the use of $\mathrm{CoSi}_{2}$ layers of increasingly small dimension. Apart from grain-boundary encrgics and kinctics, the problems encountered during growth of silicides require a better understanding of both the surface and interface free energies. This is especially important for very thin films or layers subjected to high-temperature anneals where each grain independently contributes to the overall film morphology.

The role of the interfacial and surface energetics is made clear by describing stable equilibrium for a particle located on a forcign substrate. As outlined by several authors, ${ }^{7-9}$ an arbitrarily shaped precipitate attached to a surface will change its shape upon annealing to minimize the Helmholtz free energy of the system, $\int \gamma(\theta) d A$. At the triple point, defined as the point at which the crystalline substrate, precipitate, and vacuum intersect, equilibrium is characterized by the total free energy being stationary with respect to infinitesimal displacements. Commonly, crystalline solids are assumed to follow the liquid drop approximation $[\gamma(\theta)=$ const $]$ in order to extract some measure of the energies involved from a simple line tension balance; ${ }^{10}$ however, this is not a rigorously correct assumption. Stable equilibrium for isolated, crystalline particles (such as $\mathrm{CoSi}_{2}$ in this study) is more completely determined by considering the anisotropy of the free-energy terms, where

$$
\begin{aligned}
\sum_{i=1}^{3} \int \gamma_{i}(\theta) d A_{i}= & \int \gamma_{s-v}(\theta) d A_{1}+\int \gamma_{s-p}(\theta) d A_{2} \\
& +\int \gamma_{p-v}(\theta) d A_{3}
\end{aligned}
$$

is stationary, or ${ }^{7}$

$$
\sum_{i=1}^{3}\left(\gamma_{i} l_{i}+\frac{\partial \gamma_{i}}{\partial t_{i}}\right)=0
$$

Hence, the problem of describing equilibrium for a crystalline particle on a surface requires knowledge about each of the surface tension terms $\gamma_{i}$ and the corresponding step energies taken from $d \gamma_{i} / d t_{i}$ near a cusp. In this formulation, $\gamma_{s-v}, \gamma_{p-v}$, and $\gamma_{s-p}$ are the substrate surface free energy, the particle surface free energy, and the substrate-particle interfacial free energy, respectively.

While the equilibrium shape of Si (i.e., $\gamma_{s-v}$ ) has been determined previously and the corresponding step energies $(\partial \gamma / \partial t)$ have been measured, ${ }^{11}$ little is known about the interfacial and surface free energy of $\mathrm{CoSi}_{2}$. Therefore, in this study we attempt to characterize the energetics of $\mathrm{CoSi}_{2}$ on $\mathrm{Si}$. This has been accomplished experimentally by studying the shapes of $\mathrm{CoSi}_{2}$ particles which form at or are buried 
below the Si surface. Studies of small, isolated volumes of silicide (formed from a $2 \AA$ layer of $\mathrm{Co}$ ) allow us to monitor the energetics by reducing the role of long range diffusion. "Buried precipitates" (i.e., CoSi $\mathrm{CO}_{2}$ equilibrated within the Si lattice) are used to provide information about the $\mathbf{S i}$ precipitate interfacial energy $\gamma_{s-p}$. Ratios of $\gamma_{s-p}$ for different low-energy orientations are then extracted using a reverse Wulff construction instead of a simple line tension balance. "Surface precipitates" (equilibrated at the $\mathrm{Si}$ surface) are used to gauge which crystallographic planes are lowest in free energy $\gamma_{p-v}$, for silicide equilibrated in vacuum.

\section{EXPERIMENT}

$\mathrm{CoSi}_{2}$ was grown and equilibrated on clean, reconstructed $\mathrm{Si}\langle 100\rangle$ substrates in an ultrahigh-vacuum (UHV) environment. ${ }^{12}$ The first step in this process involved preparing $\mathrm{Si}(100\rangle$ substrates outside the vacuum chamber using a modified RCA clean. ${ }^{13}$ A protective oxide was grown at the end of this procedure. Samples were then loaded onto detachable cassettes, placed into the chamber apparatus via a load-lock chamber, and outgassed thoroughly in a preparation chamber $\left(150^{\circ} \mathrm{C}\right.$ for $1 \mathrm{~h}, 250^{\circ} \mathrm{C}$ for $1 \mathrm{~h}, 600^{\circ} \mathrm{C}$ for $2-3$ h). After insertion into the analysis chamber, the protective oxide was removed by heating to $950^{\circ} \mathrm{C}$ for $1 \mathrm{~min}$, and $350-\AA$-thick undoped Si buffer layers were grown $(0.4 \AA / \mathrm{s}$, $\left.T \sim 660-700{ }^{\circ} \mathrm{C}\right) .2 \AA$ of cobalt was then deposited using an electron-beam evaporator with the substrates heated to $400{ }^{\circ} \mathrm{C}$. Afterward, samples were either annealed to high temperatures (forming surface precipitates) or capped with epitaxial Si and then annealed (to equilibrate buried precipitates). In both cases, the shape equilibration step consisted of heating to $950^{\circ} \mathrm{C}$ for several hours. Also, several samples were annealed to $1050^{\circ} \mathrm{C}$ for $2 \mathrm{~h}$. No difference in shape was found for samples annealed at $950^{\circ} \mathrm{C}$ compared with those annealed to higher temperatures (Note, the melting temperature of $\mathrm{CoSi}_{2}$ has been determined to be $1600 \mathrm{~K}$.) ${ }^{14}$ Pressures in the chamber during annealing remained below $3 \times 10^{-9}$ Torr, Auger electron spectroscopy and low-energy electron diffraction were both used to ensure that clean, wellordered surfaces were used.

After removal from the vacuum system, plan-view transmission electron microscopy (TEM) samples were made by back-thinning 3-mm-diam disks to a thickness of $175 \mu \mathrm{m}$, followed by etching to perforation with a $\mathrm{CH}_{3} \mathrm{COOH}: \mathrm{HNO}_{3}: \mathrm{HF}$ (3:5:3) mixture. Cross-section TEM foils of each sample were also prepared. This procedure consisted of polishing to $50 \mu \mathrm{m}$ followed by ion milling with a $4 \mathrm{kV}$ Ar beam. TEM foils were never heated above $100^{\circ} \mathrm{C}$ during preparation. Samples were analyzed using both Philips $420 \mathrm{~T}(120 \mathrm{kV})$ and JEOL $4000 \mathrm{EX}(400 \mathrm{kV})$ electron microscopes.

\section{RESULTS}

$\mathrm{CoSi}_{2}$ precipitates grown using the procedure outlined above were found to nucleate on clean, dimerized $\mathrm{Si}\{100\}$ with one of three orientations relative to the Si surface normal. The shapes of the surface precipitates with these orientations remained stable upon annealing and are shown in a

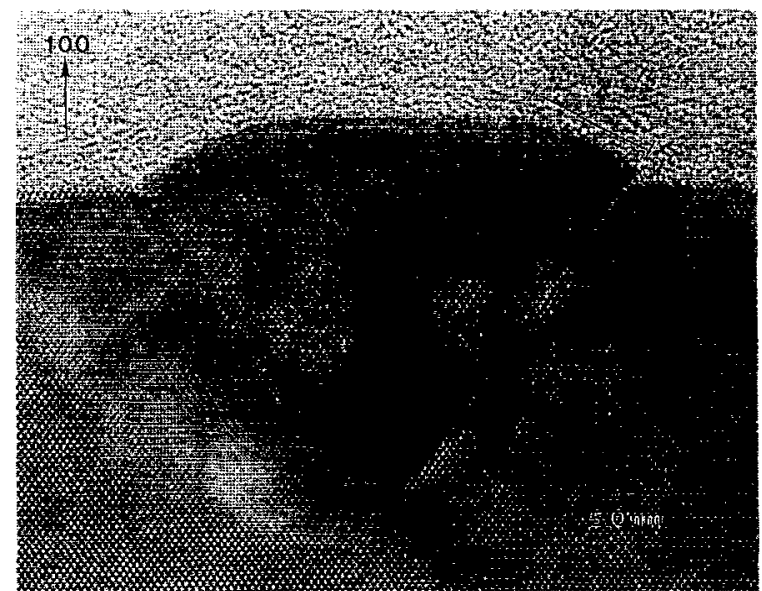

FIG. 1. Cross-section TEM image of the equilibrium shape of a surface $\mathrm{CoSi}_{2}$ precipitate with the [100] parallel to the [100] of Si. This is a $\langle 011\rangle$ projection for both $\mathrm{Si}$ and $\mathrm{CoSi}_{2}$. " $\mathrm{A}$ " marks the length of the curved region.

number of cross-section transmission electron micrographs. Each high-resolution TEM image described below is aligned along a $\langle 011\rangle$ projection for $\mathrm{Si}$. Figure 1 shows the shape of the largest fraction of $\mathrm{CoSi}_{2}$ surface precipitates found in this study. In fact, on several substrates $-95 \%$ of all precipitates formed with this shape (see the plan-view TEM image and corresponding diffraction pattern in Fig, 2). These epitaxial precipitates were oriented such that

$$
[100]_{\mathrm{CoSi}_{2}} \|[100]_{\mathrm{Si}}
$$

and

$$
[011]_{\mathrm{CoSi}_{2}} \|[011]_{\mathrm{Si}}
$$

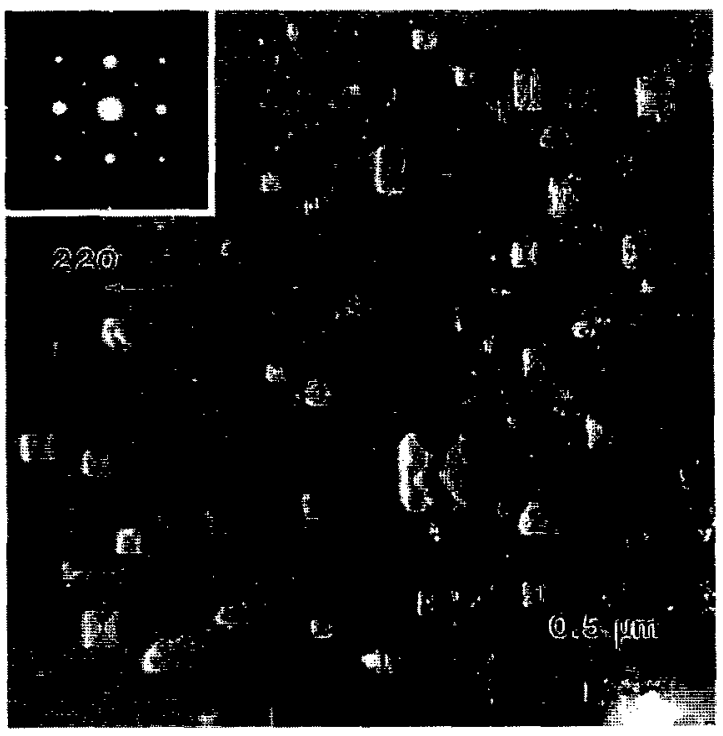

FIG. 2. Plan-view TEM weak-beam image $(g=220)$ showing the equilibrium shape of surface $\mathrm{CoSi}_{2}$ precipitates, mostly with the [100] paraliel to the $[100]$ of $\mathrm{Si}$. 


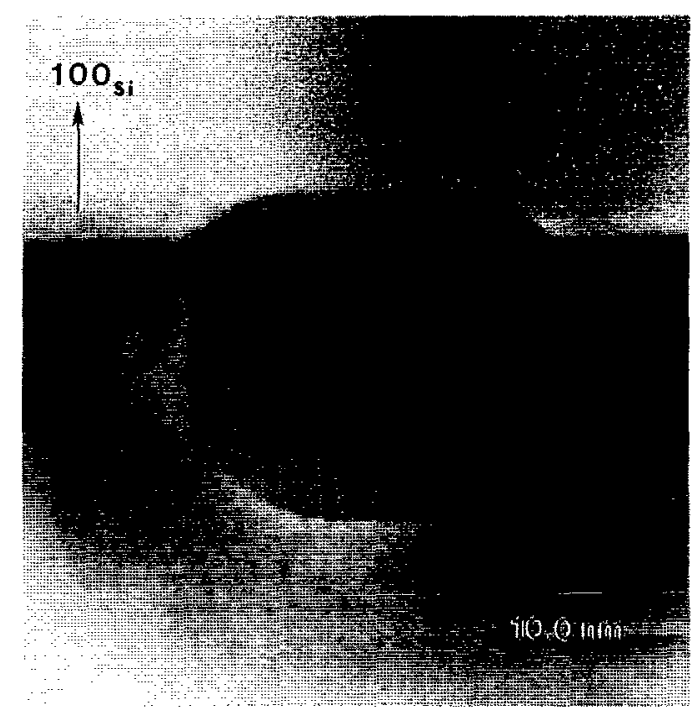

FIG. 3. Cross-section TEM image of the equilibrium shape of a surface $\mathrm{CoSi}_{2}$ precipitate with the [110] parallel to the [100] of Si shown. $\langle 011\rangle$ projection for $\mathrm{Si}$.

This precipitate formed interfaces mostly along $\{111\}$ and $\{100\}$ planes. Above the $\mathrm{Si}$ surface, the $\mathrm{CoSi}_{2}$ particle was facetted on a (100) plane, away from the triple point. The area of this top (100) facet was found to scale roughly with the size of the precipitate, where the curved region near the triple point did not extend to lengths greater than $\sim 75 \AA$, as illustrated in Fig. 1.

The other surface precipitate shape often found on $\mathrm{Si}\{100\}$ substrates is shown in Fig. 3. The different orientation of this equilibrated $\mathrm{CoSi}_{2}$ particle is indicated by the diffraction pattern inset in Fig. 4. These particles were aligned such that $\langle 110\rangle$ of $\mathrm{CoSi}_{2}$ was parallel to the [100] of Si. Two variations of this $\langle 110\rangle$ alignment were found, as

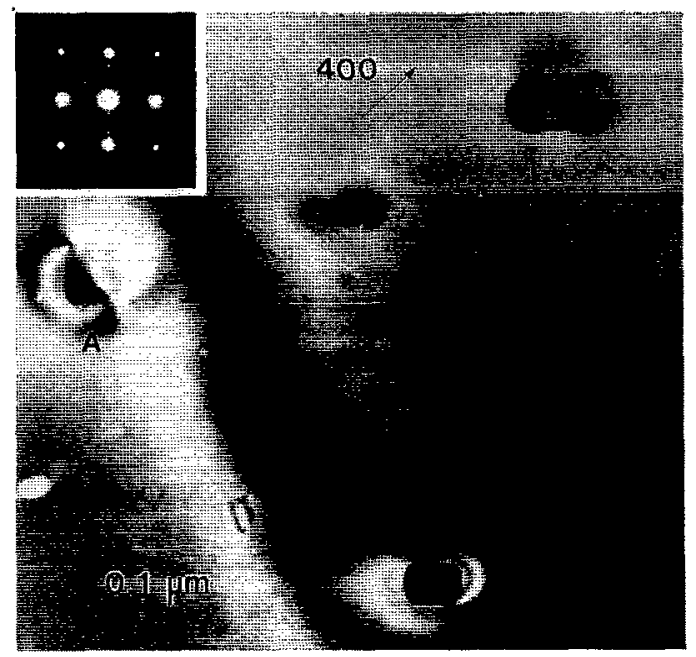

FIG. 4. Plan-view TEM bright-field image $(g=400)$ of the equilibrium shape of surface $\mathrm{CoSi}_{2}$ precipitates, mostly with the [110] parallel to the $[100]$ of $\mathrm{Si}$.

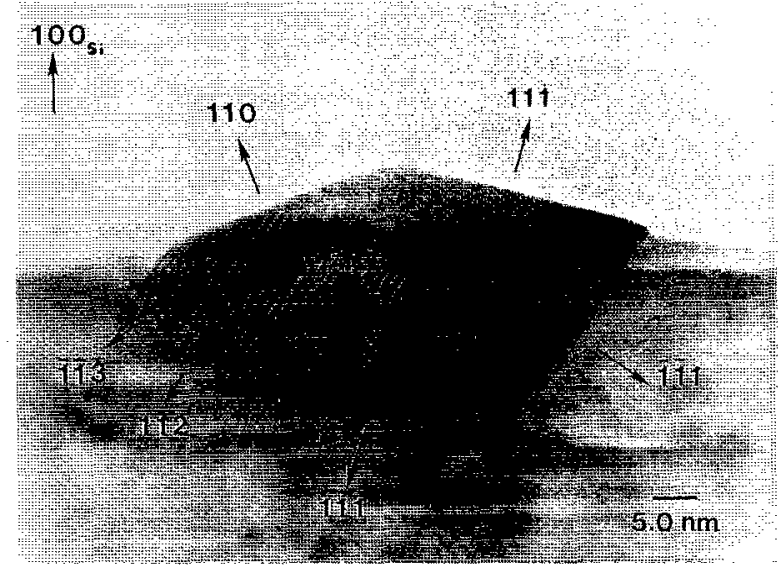

FIG. 5. Cross-section TEM image of the equilibrated shape of a surface $\mathrm{CoSi}_{2}$ precipitate with $[221]_{\mathrm{CoSi}}$, parallel to the [100] $]_{\mathrm{Si}}$ shown in the upper left-hand-side corner. All other labels identify directions of the silicide along which facets have formed. $\langle 011\rangle$ projection for $\mathrm{Si}$.

with previous work. ${ }^{15}$ One variation included $\mathrm{CoSi}_{2}(110)$ on Si(100) with

$$
[011]_{\operatorname{CoSi}_{2}} \|[100]_{\mathrm{Si}}
$$

and

$$
[011]_{\mathrm{CoSi}_{2}} \|[011]_{\mathrm{Si}} \text {. }
$$

The other variant of the $\mathrm{CoSi}_{2}(110)$ precipitates was oriented with

$$
[011]_{\operatorname{Cos}_{2}} \|[100]_{\mathrm{Si}}
$$

and

$$
[01 \overline{1}]_{\mathrm{CoSi}_{2}} \|[01 \overline{1}]_{\mathrm{Si}} \text {. }
$$

TEM revealed that the shapes of the two variants of (110)oriented precipitates were the same (see Fig. 4). All (110)oriented precipitates formed with a shape having a welldefined aspect ratio, when imaged in plan view. The projected "sides" of these precipitates are triple-point edges lying mostly along [110] and [100]. Consistently, this aspect ratio was measured along $\langle 110\rangle_{\mathrm{CoSi}_{2}}\left\langle\langle 100\rangle_{\mathrm{CoSi}_{2}}\right.$ to be equal to 1.37. This was the case for both variants. As can be seen in Fig. 4, the precipitate labeled $A$ is extended in a direction $90^{\circ}$ from the longer direction for each of the other precipitates shown. This corresponds to the $\langle 01 \overline{1}\rangle$ for the second variant. The portion of the (110) surface precipitates equilibrated within the Si always formed with a rounded shape (see Fig. 3).

A small number of precipitates aligned with the $[221]_{\mathrm{CoSi}_{2}}$ parallel to the $[100]_{\mathrm{Si}}$ surface normal were also found. ${ }^{16}$ These precipitates were stable upon annealing and took the shape shown in Fig. 5. This $\mathrm{CoSi}_{2}$ precipitate was not symmetrical but formed a number of facets of different lengths. Above the Si surface, this precipitate had both (111) and (110) surfaces. The areas of these particular facets were approximately the same in all samples, showing no prefer- 


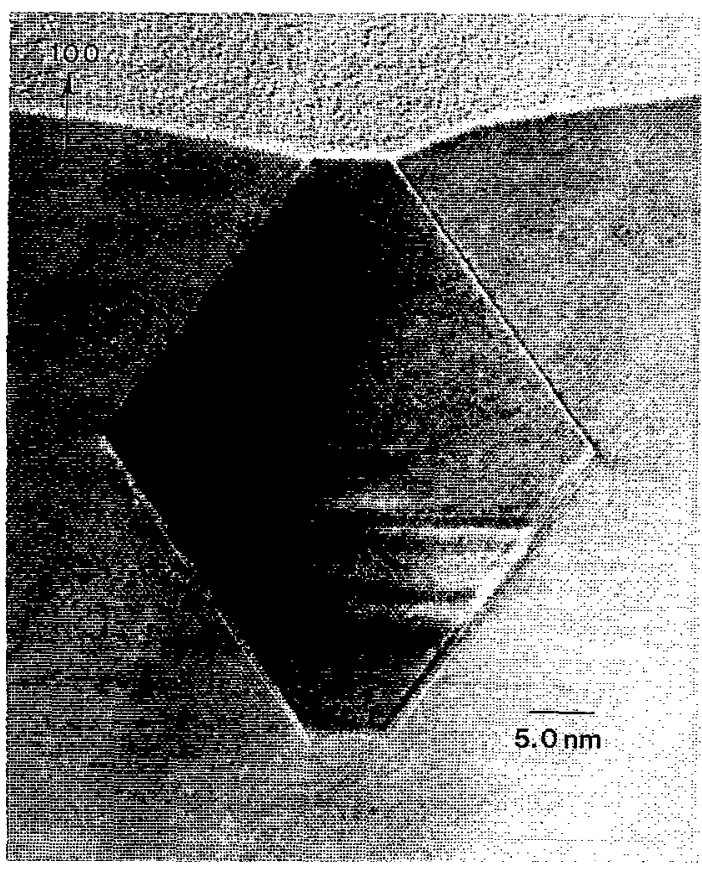

FIG. 6. Cross-section TEM image of the equilibrium shape of a buried $\mathrm{CoSi}_{2}$ precipitate with the $[100]$ parallel to the $[100]$ of $\mathrm{Si}$.

ence to form along one particular orientation. A number of interfaces formed below the Si surface. These are also indexed in Fig. 5.

$\mathrm{CoSi}_{2}$ precipitates equilibrated entirely within Si displayed shapes similar to the buried portions of surface precipitates. In this study, the shape of [100]-oriented buried precipitates were analyzed. The shapes of these precipitates were always as shown in Fig. 6. Note the particular precipitate shown in Fig. 6, happened to break through to the Si surface, but still displays the shape found for [100]-oriented buried precipitates. These developed $\{111\}$ and $\{100\}$-faceted interfaces with Si. Again, no $\{110\}$-faceted interfaces were observed. These particles have been used to monitor the interfacial free energy of $\mathrm{CoSi}_{2}$ in $\mathrm{Si}$. The relative interfacial free energies of the two low-energy orientations were estimated using a reverse Wulff approach. A "center point" was fixed for each and radii were drawn to the respective facet planes of the equilibrium shape. ${ }^{7}$ Plots of radius as a function of crystallographic direction, such as that in Fig. 7, provided the ratio of

$$
r\{100\} / r\{111\}=\gamma\{100\} / \gamma\{111\}=1.43 \pm 0.07 .
$$

\section{DISCUSSION}

It is evident that the interfacial energy of the $\mathrm{CoSi}_{2}-\mathrm{Si}$ system dominates the morphology of $\mathrm{CoSi}_{2}$ films grown on $\mathrm{Si}$ in this manner. The silicide does not wet the Si surface, but forms by digging into the Si. The process of annealing isolated silicide precipitates for long times has also shown that a number of common features are present, despite the fact that isolated $\operatorname{CoSi}_{2}$ precipitates nucleated with several different orientations. In particular, the $\{111\}$, and to a lesser degree $\{100\}, \mathrm{CoSi}_{2}-\mathrm{Si}$ interfaces were consistently found to

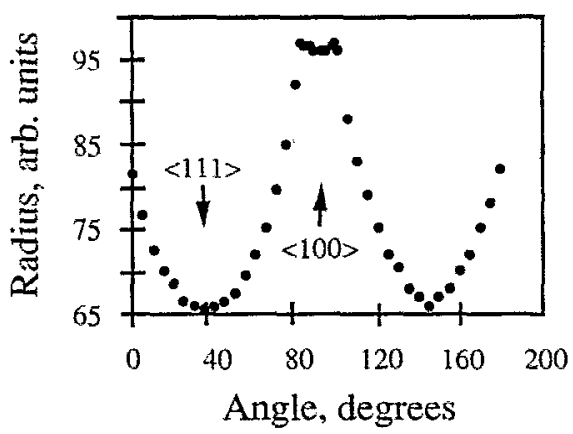

FIG. 7. Plot of radius vs misorientation angle for equilibrated buricd $\mathrm{CoSi}_{2}$ precipitate.

form. Evidence of this was seen in both the symmetrical precipitates surrounded entirely by $\mathrm{Si}$ and the buried portions of surface precipitates. Of these two crystallographic planes, the $\{111\}$ dominates the equilibrium shape. This preference to form along $\{111\}$ was identified previously in work involving channeled implantation of Co followed by rapid thermal annealing for brief amounts of time. ${ }^{17}$ In this study, much longer anneals have provided an accurate measure of the relative magnitudes of the $\{111\}$ and $\{100\}$ interfacial free energies. The assumption that equilibrium has been established is made because all buried $\mathrm{CoSi}_{2}$ particles with this particular orientation maintained the same shape, independent of anneal time and temperature for the ranges tested. Also, the shape remained constant for particles of different size. The difference in the [100] shape here and the variety of shapes found previously involving a rapid thermal anneal is most likely explained by the change in annealing time (i.e., hours compared to times $-30 \mathrm{~s}$ ). ${ }^{17}$ The reverse Wulff construction applied to a large number of particles in this study showed that the $\{111\}$ has the lower energy, with ratios of $r\{100\} / r\{111\}$ indicating that $\left.\gamma^{\prime} 100\right\} / \gamma\{111\}=1.43 \pm 0.07$.

To our knowledge, a theoretical study has not yet predicted this ratio. While Hamann has employed the localdensity-functional method ${ }^{18}$ in the past for calculating the $\mathrm{CoSi}_{2}\{111\}$-Si $\{111\}$ interfacial energies for both the A- and B-type interfaces, ${ }^{6,19,20}$ a similar result is needed for the $\{100\}$ interface. Past work on extracting energies for the \{111\} B-type interface did conclude that the eightfold coordinated Co structure has the lowest energy, ${ }^{18,21}$ with an energy of $0.53 \mathrm{eV} .^{18}$ Work using TEM has since verified the eightfold coordination of $\mathrm{Co}$ atoms at the B-type interface. ${ }^{22}$ Additional work has suggested that $\mathrm{Co}$ atoms located at the $\mathrm{CoSi}_{2}\{111\}$-Si $\{111\}$ A-type interface are either sevenfold ${ }^{23,24}$ or eightfold coordinated. ${ }^{25}$ In this study, we have found that both A- and B-type interfaces form on the annealed precipitates. The equilibrium shape shown in Fig. 6 (used for extracting a measure of interfacial free energies) has A-type $\{111\}$ intertaces. The $\mathrm{CoSi}_{2}$-Si interface, labeled as [ii1] in Fig. 5, is B type. The [221]-oriented surface precipitates further indicate that the larger propensity to form interfaces along $\operatorname{CoSi}_{2}\{111\}$ planes is independent, to some degree, of the alignment to the Si. These precipitates showed that $\mathrm{CoSi}_{2}\{111\}$ planes formed interfaces with different $\mathrm{Si}\{h k l\}$ 
planes, including both $\mathrm{Si}\{111\}$ (labeled as [ī11]) and close to $\mathrm{Si}\{511\}$ planes (labeled as [i $\overline{1} \overline{1}]$ ).

Comparison of the many precipitate shapes has indicated that the surface energetics of $\mathrm{CoSi}_{2}$ are not identical to the interfacial energetics. The difference in these two equilibrium shapes involves the formation along $\operatorname{CoSi}_{2}\{110\}$ planes. The $\mathrm{CoSi}_{2}$-Si interfaces formed in each case did not include (110). On the other hand, the portions of $\mathrm{CoSi}_{2}$ precipitates forming above the Si surface (away from the triple points) suggest that the equilibrium shape of $\mathrm{CoSi}_{2}$ in vacuum contains significant portions of (100), (111), and (110). Large (110) facets are found for all (110)-oriented surface precipitates, and for the $\mathrm{CoSi}_{2}$ precipitates aligned with the [221] parallel to the Si surface normal direction. Nevertheless, the full equilibrium shape for $\mathrm{CoSi}_{2}$ surface free energy has not been found. It is expected that other low-energy $\mathrm{CoSi}_{2}$ surfaces and $\mathrm{CoSi}_{2}$-Si interfaces may exist even though they do not appear on the symmetrical precipitates analyzed in this study. For example, the [221]-oriented surface precipitates did develop smaller buried facets along $(\overline{1} \overline{1} \overline{2})$ and $(\overline{1} \overline{1} \overline{3})$ of $\mathrm{CoSi}_{2}$. In this comparison of buried and surface $\mathrm{CoSi}_{2}$ precipitates, we have assumed that interfacial and surface energetics play the dominant role in shape formation; however, we recognize that other energetic factors (e.g., strain energy) may have an effect on shape.

It is certain that both the surface and interfacial free energies will act as thermodynamic driving forces to influence the morphology of a $\mathrm{CoSi}_{2}$ layer, especially when using a salicide process. Therefore, future use of $\mathrm{CoSi}_{2}$ layers of increasingly small dimension will require modification of the growth process. The Si- silicide interfacial roughness for films grown on $\langle 100\rangle$ substrates using a salicide process apparently could benefit from a reduction of the $\{100\}$ interfacial free energy at the cxpense of the $\{111\}$. Already, initial work aimed at increasing stability has been attempted. The introduction of foreign species via implantation has been used on both polycrystalline $\mathrm{TiSi}_{2}$ and $\mathrm{CoSi}_{2}$ layers. ${ }^{26} \mathrm{Ac}-$ cording to Wang et al., the addition of $\mathrm{F}$ improves the quality of the silicide layer, because $\mathrm{F}$ is accumulated at the silicide grain boundaries and at the silicide/silicon interface. ${ }^{27} \mathrm{Nev}$ ertheless, the degree to which this species modifies the energetics is not known. A more accurate determination of the surface energetics involving an equilibrium-shape-type analysis is needed before the role of $F$ on the surface free energy can be determined. Also, a better understanding of the nucleation stage of growth is needed in order to successfully apply a salicide process to the growth of stable $\mathrm{CoSi}_{2}$ layers. In this study, we have found that isolated $\mathrm{CoSi}_{2}$ precipitates form on the $\mathrm{Si}\{100\}$ surface with one of several different orientations that remain stable upon annealing to high temperatures. The formation of both [110]- and [221]misoriented grains has been explained in terms of the initial Si surface topography. ${ }^{5,16}$ In particular, the step structure of the $\{100\}$ surface and the technique used to grow a silicide layer were found to affect the resultant silicide orientation.

\section{SUMMARY}

The energetics associated with $\mathrm{CoSi}_{2}$ grown onto a $\mathrm{Si}$ surface have been analyzed by studying $\mathrm{CoSi}_{2}$ equilibrated below and above the $\mathrm{Si}$ lattice. $\mathrm{CoSi}_{2}$ precipitates buried within the Si lattice form preferentially along $\{111\}$ and $\{100\}$ planes. Using a reverse Wulff approach instead of a line tension balance, the ratio of interfacial free energies of $\chi\{100\} / \chi\{111\}$ was measured to be $1.43 \pm 0.07$. In addition, a difference in the surface energetics compared with the interfacial energetics has been detected. The shape of equilibrated silicide surface precipitates suggests that the orientations of $\mathrm{CoSi}_{2}$ with low surface free energy are $\{111\},\{100\}$, and $\{110\}$. A basis is now established for identifying how the energetics are being influenced by changing the growth process.

\section{ACKNOWLEDGMENT}

This work was supported in part by the NSF under Contract No. DMR 9202176.

${ }^{1}$ S. P. Murarka, Silicides for VLSI Applications (Academic, New York, 1983).

${ }^{2}$ J. M. Phillips, J. L. Batstone, J. C. Hensel, M. Cerullo, and F. C. Unterwald, J. Mater. Res. 4, 144 (1989).

${ }^{3}$ Z. G. Xiao, G. A. Rozgonyi, C. A. Canovai, and C. M. Osburn, J. Mater. Res. 7, 269 (1992).

${ }^{4}$ W. W. Mullins, J. Appl. Phys. 28, 333 (1957).

${ }^{5}$ S. M. Yalisove, R. T. Tung, and D. Loretto, J. Vac. Sci. Technol. A 7, 1472 (1989).

${ }^{6}$ R. T. Tung, J. L. Batstone, and S. M. Yalisove, J. Electrochem. Soc. 136, 815 (1988)

${ }^{7} \mathrm{C}$. Herring, Structure and Properties of Solid Surfaces (University Press, Chicago, 1953), p. 5-72.

8 J. W. Gibbs, Collected Works (Longman, Green, New York, 1928).

${ }^{9}$ W. W. Mullins, in Metal Surfaces: Structure, Energetics and Kinetics, edited by Robertson and Gjostein (American Society of Metals, Metals Park, OH, 1963), p. 170.

${ }^{10}$ H.-T. Jeon, R. J. Nemanich, J. W. Honeycutt, and G. A. Rozgonyi, Mater. Res. Soc. Symp. Proc. 160, 307 (1990).

${ }^{11}$ D. J. Eaglesham, A. E. White, L. C. Feldman, N. Moriya, and D. C. Jacobson, Phys. Rev. Lett. 70, 1643 (1993).

${ }^{12}$ S. M. Yalisove, D. P. Adams, and O. P. Karpenko (unpublished).

${ }^{13}$ A. Ishizaka and Y. Shiraki, J. Electrochem. Soc. 133, 666 (1986).

${ }^{14}$ M. Nakamura, Met. Mater. Trans. A 25, 331 (1994).

${ }^{15}$ S. M. Yalisove, R. T. Tung, and J. L. Batstone, Mater. Res. Soc. Symp. Proc. 116, 439 (1988).

${ }^{16}$ C. W. T. Bulle-Lieuwma, A. H. van Ommen, and J. Hornstra, Mater. Res. Soc. Proc. 102, 377 (1988).

${ }^{17}$ K. Radermacher, S. Mantl, K. Kohlohof, and W. Jager, J. Appl. Phys. 68, 3001 (1990).

${ }^{18}$ D. R. Hamann, Phys. Rev. Lett. 60, 313 (1988).

${ }^{19}$ R. T. Tung, J. M. Poate, J. C. Bean, J. M. Gibson, and D. C. Jacobson, Thin Solid Films 93, 77 (1982).

${ }^{20}$ R. T. Tung, J. M. Gibson, J. C. Bean, J. M. Poate, and D. C. Jacobson, Appl. Phys. Lett. 40, 684 (1982).

${ }^{21}$ P. J. van den Hoek, W. Ravenek, and E. J. Barends, Phys. Rev. Lett. 60, 1743 (1988).

${ }^{22}$ C. W. T. Bulle-Lieuwma, A. F. de Jong, A. H. van Ommen, J. F. van der Veen, and J. Vrijmoeth, Appl. Phys. Lett. 55, 648 (1989).

${ }^{23}$ A. F. de Jong and C. W. T. Bulle-Lieuwma, Philos. Mag. A 62, 183 (1990).

${ }^{24}$ C. W. T. Bulle-Lieuwma, A. H. van Ommen, and L. J. van Ijzendoom, Appl. Phys. Lett. 54, 244 (1989).

${ }^{25}$ M. F. Chisholm, S. J. Pennycook, R. Jebasinski, and S. Mantl, Appl. Phys. Lett. 64, 2409 (1994).

${ }^{26}$ A. Nishiyama, Y. Akasaka, Y. Ushiku, K. Hishioka, Y. Suizu, and M. Shiozaki, in Proceedings of the 7th International IEEE VLSI Multilevel Interconnection Conference (VMIC), Santa Clara, CA, 1990, p. 310.

${ }^{27}$ Q. F. Wang, J. Y. Tsai, C. M. Osburn, R. Chapman, and G. E. McGuire, Appl. Phys. Lett. 61, 2920 (1992). 